\title{
RADIOGRAFÍA DE LA ESTRUCTURA LABORAL TRAS LOS GOBIERNOS KIRCHNERISTAS
}

\author{
Radiography of the labor structure after the kirchner goverments
}

Ianina Harari ${ }^{1}$

Nicolás Villanova ${ }^{2}$

Resumen

Eduardo Sartelli ${ }^{3}$

Uno de los ejes de debate acerca de los gobiernos Kirchneristas, cuyo mandato se extendió entre 2003 y 2015, fue las características que adquirió el mercado de trabajo tras las políticas laborales implementadas. La dificultad del acceso a estadísticas confiables ha sido un obstáculo para la interpretación del proceso. Mediante la reconstrucción estadística a partir de los datos disponibles, se evalúa tanto la estructura laboral, así como la calidad del empleo y el nivel de afiliación sindical que resultaron de las políticas kirchneristas. Se verifica la persistencia del trabajo no registrado, un elevado porcentaje de contratos a término, la continuidad de la caída de la tasa de sindicalización, entre otros aspectos que se verán en perspectiva histórica.

\section{Palabras clave}

Trabajo; clase obrera; estructura laboral; Kirchnerismo; Argentina.

\section{Abstract}

One of the axes of debate about the Kirchnerist governments, whose mandate extended between 2003 and 2015, was the characteristics acquired by the labor market structure after the implemented labor policies. The difficulty of access to reliable statistics has been an obstacle to the interpretation of the process. By means of the statistical reconstruction based on the available data, the labor market structure, as well as the quality of the employment and the level of unionization that resulted from the Kirchner policies are evaluated. The persistence of non-registered work, a high percentage of forward contracts, the continuity of the fall in the unionization rate, among other aspects that will be seen in historical perspective, are verified.

\section{Keywords}

Work; working class; labor structure; kirchnerism; Argentine.

\footnotetext{
${ }_{1}^{1}$ Centro de Estudios e Investigaciones Laborales CONICET (Argentina). Correo electrónico: ianinaharari@yahoo.com.ar.

${ }^{2}$ Centro de Estudios e Investigación en Ciencias Sociales - CEICS (Argentina). Correo electrónico: nicovillanova@yahoo.com.ar.

${ }^{3}$ Centro de Estudios e Investigación en Ciencias Sociales - CEICS (Argentina). Correo electrónico: eduardo.sartelli@yahoo.com.ar.
} 


\section{Introducción}

Uno de los principales debates que han atravesado los estudios sobre el trabajo en la última década se centró en las características del empleo a partir de las políticas implementadas por los gobiernos kirchneristas. Los análisis acerca de los resultados de dichas políticas se ubican en torno a dos polos. Por un lado, quienes sostienen la tesis de que, junto con una recomposición cuantitativa en los niveles de empleo, se habría producido una mejora cualitativa. Por otro, se encuentran quienes critican la posición anterior y advierten que la recuperación de las cifras de ocupación, no necesariamente tuvo correlato en las condiciones de trabajo.

La visión más positiva del proceso plantea que, durante la década kirchnerista, la sociedad se volvió a vertebrar en torno al trabajo, por el crecimiento de la tasa de asalarización, y al crecimiento del empleo industrial y los servicios básicos asociados a la industria (Palomino y Dalle, 2012), dado el proceso de "sustitución de importaciones". Asimismo, se habría producido un cambio en la composición del empleo al incrementarse el peso del trabajo registrado. Todo ello implicaría un quiebre de las tendencias previas (Panigo y Neffa, 2009; Beccaria y Maurizio, 2012) y sería prueba del surgimiento de un nuevo régimen de empleo como consecuencia de la acción del Estado, que habría asumido un nuevo rol, y de la redefinición de la estrategia de los sindicatos (Palomino, 2008). Respecto a estos últimos, se ha señalado el peso que cobraron en el período como parte de lo que se llamó "revitalización sindical", siendo algunos de los indicadores de aquel fenómeno el aumento de la tasa de afiliación y de la negociación colectiva (Senén, Trajtemberg, y Medwid, 2010).

Esta posición ha sido cuestionada mediante estudios que abordan el período, desde un enfoque que considera que las políticas económicas no resultan condición suficiente para alterar la estructura económica que se corresponde a un régimen de acumulación dominante, caracterizado como heterogéneo, dual, combinado y altamente concentrado (Salvia y Gutierrez, 2013). La reducción de los niveles de desempleo existentes a fines de la década de los noventa no habría generado modificaciones sustantivas en el mercado de trabajo en cuanto a su segmentación, la inserción ocupacional y la existencia de una sobrepoblación relativa, excedentaria a las necesidades de acumulación. En relación a la producción industrial, se ha señalado que no se produjo un cambio estructural que modifique el perfil productivo moldeado en las décadas previas y que el incremento del empleo en este sector no generó una disminución del trabajo no registrado y precario (Azpiazu y Schorr, 2010).

Respecto a la distribución del empleo creado por ramas de actividad, Beccaria y Maurizio (2012) señalan que las diferencias en la dinámica sectorial del empleo no siguieron estrictamente las que se registran en el Producto Bruto Interno (PBI). Así, señalan que se observa un aumento del empleo en ramas del sector servicios, como servicios inmobiliarios, computación o seguridad, aún sin un correlato en el incremento de su nivel de actividad, mientras que los sectores de transporte y comercio perdieron importancia sobre el total, a diferencia de lo que ocurrió con sus PBI. Asimismo, señalan que la actividad manufacturera habría vivido una gran expansión, de la mano de la sustitución de importaciones, pero que su participación relativa en el empleo total no se modificó.

Por su parte, Bekerman y Vázquez (2016) analizaron la producción, productividad y empleo de los principales sectores industriales, en categorías según su nivel tecnológico y su valor agregado, y encontraron que durante el período que se denominó pos-convertibilidad se consolidaron aquellos sectores con menor nivel tecnológico. En el mismo sentido, Fernández y Porta (2008) señalan que el empleo se ha reactivado en ramas más trabajo-intensivas, sin registrarse ningún avance hacia una mayor productividad. No podría hablarse desde esta perspectiva, entonces, de un cambio estructural.

También se ha cuestionado la idea de que las políticas laborales de los gobiernos kirchneristas fueron de la mano de una revitalización sindical. El punto que nos interesa de ese debate es el de la tasa de afiliación. Atzeni y Ghigliani (2008) señalan que se mantuvo en niveles elevados, pero las cifras padecen de cierta distorsión al no reflejar la escala que adquirió el trabajo no registrado.

En este artículo proponemos abordar la situación de los trabajadores asalariados a partir de los resultados que se observan al final de los tres gobiernos kirchneristas, entendiendo que deben comprenderse como una totalidad, sobre la base de la estadística disponible, y en una perspectiva histórica que permita situar el período en el marco de tendencias generales, para establecer si las mismas fueron o no revertidas. Se comenzará con una breve reseña de las principales reformas en el campo de las políticas laborales del kirchnerismo. Luego, se pasará 
al análisis cuantitativo de ciertas variables significativas. Para ello se utilizarán, en especial, las bases de datos de la Encuesta Permanente de Hogares (EPH). Si bien la EPH presenta limitaciones, dado que releva una muestra en los principales aglomerados urbanos, es la única que resulta de utilidad para reconstruir la imagen de la estructura laboral de la clase obrera argentina. Los datos del Censo de población de 2010 son escasos y, muchos de ellos, también están basados en una muestra. Por otro lado, el último censo económico fue publicado en 2005 pero contiene datos relevados en 2003. Para la clase obrera rural no se cuenta con el Censo Agropecuario ya que el último es de 2002.

El objetivo del presente estudio es analizar la estructura de la clase obrera ocupada sobre el final del período kirchnerista, con el propósito de evaluar cuáles son las principales características del empleo en la Argentina $y$, a partir de ellas, indagar en qué medida las políticas estatales impactaron en una mejora de las condiciones laborales generales. En primera instancia se analiza la distribución por rama de actividad, y si existe vinculación con el peso de esos sectores en el conjunto de la economía. Luego, se examina el trabajo no registrado, su relación con la distribución de la fuerza de trabajo por tamaño de establecimiento y la diferencia salarial con los trabajadores registrado. Otras variables que se abordan son los contratos temporales y el nivel salarial. De todas ellas se reconstruye una evolución histórica para así poder comparar el período de los gobiernos kirchneristas con la trayectoria previa de cada uno de los indicadores. Por último, la evolución histórica de la tasa de afiliación podrá indicarnos los límites que parecen haber alcanzado lo que se denominó el proceso de "revitalización". Si bien la discusión respecto a este concepto es amplia, nos interesa particularmente analizar la tasa de afiliación, porque es un indicador del nivel de organización corporativa de la fracción ocupada de la clase obrera, y el interés por esa forma de organización en tanto órgano de defensa de sus intereses económicos. A su vez, el análisis del nivel salarial nos permitirá evaluar si el mayor dinamismo de la negociación colectiva dio como resultado una recomposición en términos históricos del nivel salarial.

\section{La regulación laboral}

Durante el período de los gobiernos kirchneristas (20032015), se dictaron una serie de normativas legales que tendieron a regular las relaciones laborales. Muchas de ellas, buscaron reducir el nivel de empleo no registrado.
En 2004, el gobierno de Néstor Kirchner presentó un proyecto de ley que fue aprobado para la derogación de la reforma laboral sancionada bajo el gobierno de De la Rúa, pero que mantenía vigente la legislación del período menemista, e introdujo algunas modificaciones (Ley 25.877/04). Entre los puntos más importantes se encontraba el tope indemnizatorio. Se mantuvo el tope del salario base sobre el que se calculan las indemnizaciones que se impuso durante los gobiernos de Menem (Ley $23.697 / 89$ y $24.013 / 91$ ), que consistía en un promedio de las remuneraciones del convenio calculado por el Ministerio de Trabajo, pero redujo el piso indemnizatorio de dos salarios a uno. Sobre las modalidades precarias de empleo, mantuvo en tres meses el período de prueba y las modalidades de pasantías.

En cuanto a la promoción del empleo y el blanqueo, se redujeron los aportes para el registro de trabajadores de pymes y grandes empresas. En 2014 se aprobó la Ley de Promoción del Trabajo Registrado y Prevención del Fraude Laboral (Ley 26.940/14), mediante la cual se eliminaban las cargas patronales relativas a las jubilaciones y las asignaciones familiares para quienes incorporasen personal, lo cual habilitaba también el blanqueo de los trabajadores no registrados. Según el tamaño del establecimiento, el empleador obtenía durante dos años un descuento para los nuevos trabajadores contratados por tiempo indeterminado. Las empresas con menos de 15 empleados no abonaban esas cargas durante el primer año y durante el segundo pagaban sólo el 25\%. Los establecimientos entre 16 y 80 trabajadores abonaban el $50 \%$ de las cargas por dos años y los de más de 80 empleados pagaban el 75\%, también por dos años. Un capítulo especial merecieron las pymes de hasta cinco empleados, a quienes se les rebajaba un 50\% las cargas por todos sus empleados, aunque se contemplaba la posibilidad de que la empresa contratase dos trabajadores más, extendiendo así este grupo al tope a siete. Además, se preveía establecer montos máximos para el pago de aseguradora de riesgo de trabajo (ART) en estas microempresas.

Otra iniciativa presente en la Ley 26.940, era la intención de extender los convenios de corresponsabilidad gremial. Estos convenios son acordados entre el sindicato y las asociaciones patronales, y permiten la sustitución de las cargas patronales normales por un mecanismo sui generis de pago diferido de una "tarifa sustitutiva", denominada así porque reemplaza los aportes empresarios. La tarifa se negocia entre el sindicato y la cámara patronal de cada sector y se actualiza periódicamente. La tarifa se establece por la unidad de producción correspondiente a cada 
actividad, por ejemplo, tonelada o quintales, y se paga una vez terminado el ciclo productivo, cuando el empresario vende su mercancía. Por tanto, se calcula en función de lo que produjo en la campaña de ese año, con la posibilidad de abonarla en cuotas. Este sistema ya era utilizado en las actividades agropecuarias y se proponía expandirlo a actividades por fuera del ámbito rural.

En relación a los trabajadores rurales, se sancionó el Régimen de Trabajo Agrario (Ley 26.727) que reemplazaba la reglamentación anterior. Esta nueva norma mantuvo al trabajador rural por fuera de le Ley de Contrato de Trabajo. Se creó la figura del trabajador permanente discontinuo, que comprendía a los trabajadores temporarios que eran contratados por un mismo empleador de manera consecutiva en más de una oportunidad para tareas estacionales o cíclicas. Con esta categoría le correspondían iguales derechos que de los trabajadores permanentes. Pero a su vez, se habilitó la posibilidad de que quienes eran empleados bajo la forma de trabajadores permanentes durante buena parte del año fueran inscritos bajo esta categoría. Otro punto destacado fue el reemplazo del Registro Nacional de Trabajadores Rurales y Empleadores (RENATRE) por el Registro Nacional de Trabajadores y Empleadores Agrarios (RENATEA). El nuevo organismo mantenía las funciones del anterior, pero pasaba a funcionar bajo la órbita del Ministerio de Trabajo. Estaba compuesto por cuatro miembros en representación del sindicato obrero con personería gremial (UATRE) y cuatro de los empleadores, y en el nuevo esquema se agregaba autoridades del Ministerio de Trabajo y representantes de los Ministerios de Economía, Agricultura y Ciencia y Tecnología. El ente se erige para la fiscalización laboral y su principal función consiste en la expedición de la libreta del trabajador y el control del cumplimiento de las obligaciones entre las partes.

\section{Distribución de la fuerza de trabajo por rama económica}

La distribución por sectores económicos, como se observa en el tabla 1, muestra que el grueso de los asalariados, más de tres cuartos, se ocupa en el sector terciario (75,9\%). Solo un $22,2 \%$ se emplea en el sector secundario. La industria manufacturera ocupa un $13,9 \%$, pero en su interior se agrupa una gran cantidad de ramas (40 en total). Cada rama económica dentro de la industria manufacturera ocupa una pequeña fracción de asalariados: ninguna de ellas supera el 1,6\% del total de ocupados. Alimentación
$(1,6 \%)$ y Metalurgia (1,5\%) lideran el ranking. El resto no alcanza siquiera al $1 \%$ del total de los asalariados. La Construcción, un 7,4\%, siendo uno de los sectores que presenta una concentración elevada de asalariados en sí mismo. Por último, un $1 \%$ se emplea en actividades primarias (agropecuarias y extractivas).

Tabla 1. Distribución porcentual de asalariados y porcentaje de PBI por sector, 2015

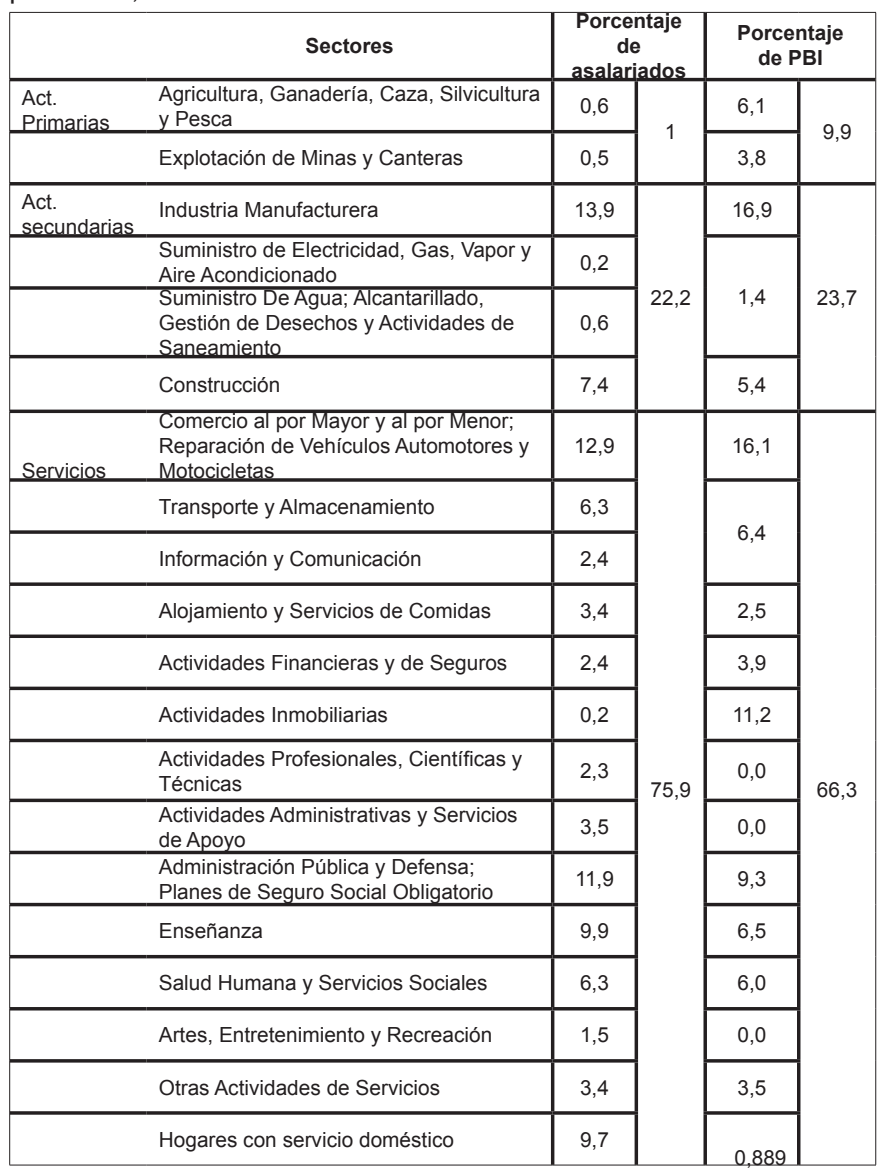

Fuente: medición propia con base en EPH y Cuentas Nacionales, INDEC.

Si comparamos esta distribución con la que existía durante los 90 vemos algunas diferencias, como observamos en la tabla 2. Tomamos como año de referencia 1995, a mediados del mandato menemista.

Se observa que, trascurridas dos décadas, el trabajo en actividades primarias mantuvo un peso similar en la estructura laboral, mientras las actividades secundarias perdieron peso en favor de las terciarias. En Particular, la industria manufacturera disminuyó su peso en un $5 \%$, la construcción subió un $2,5 \%$, comercio subió $1 \%$, transporte $2 \%$, actividades inmobiliarias casi $5 \%, y$ servicio doméstico disminuyó $1,3 \%$. 
Tabla 2. Distribución porcentual de asalariados por sector, 1995.

\begin{tabular}{|c|c|c|c|}
\hline & Sectores & \multicolumn{2}{|c|}{$\%$ de asalariados } \\
\hline \multirow{2}{*}{ Act. Primarias } & $\begin{array}{l}\text { Agricultura, Ganadería, Caza, Silvicultura y } \\
\text { Pesca }\end{array}$ & \multirow{2}{*}{1,06} & \multirow{2}{*}{1,06} \\
\hline & Explotación de Minas y Canteras & & \\
\hline \multirow{4}{*}{$\begin{array}{l}\text { Act. } \\
\text { secundarias }\end{array}$} & Industria Manufacturera & & \multirow{4}{*}{25,02} \\
\hline & $\begin{array}{l}\text { Suministro de Electricidad, Gas, Vapor y Aire } \\
\text { Acondicionado }\end{array}$ & \multirow{2}{*}{1,22} & \\
\hline & $\begin{array}{l}\text { Suministro De Agua; Alcantarillado, Gestión de } \\
\text { Desechos y Actividades de Saneamiento } \\
\end{array}$ & & \\
\hline & Construcción & & \\
\hline \multirow{10}{*}{ Servicios } & $\begin{array}{l}\text { Comercio al por Mayor y al por Menor; } \\
\text { Reparación de Vehículos Automotores y } \\
\text { Motocicletas }\end{array}$ & 11.89 & \multirow{10}{*}{73,91} \\
\hline & Transporte y Almacenamiento & 8.44 & \\
\hline & Alojamiento y Servicios de Comidas & 3.37 & \\
\hline & Actividades Financieras y de Seguros & 3,31 & \\
\hline & Actividades Inmobiliarias & 515 & \\
\hline & \begin{tabular}{|l|} 
Administración Pública y Defensa; Planes de \\
Seguro Social Obligatorio \\
\end{tabular} & 10,45 & \\
\hline & Enseñanza & 9.52 & \\
\hline & Salud Humana y Servicios Sociales & 6.04 & \\
\hline & Otras Actividades de Servicios & 467 & \\
\hline & Hogares con servicio doméstico & & \\
\hline
\end{tabular}

Fuente: medición propia con base en EPH

La distribución de la fuerza de trabajo no parece guardar relación con la participación de cada sector en la producción total, como se ve en la tabla 1. Más bien se relaciona con el proceso de trabajo que rige en cada rama. En términos marxistas, los distintos modos de organizar el proceso de trabajo son las formas que históricamente el capital ha desarrollado para aumentar la fuerza productiva del trabajo con el fin de obtener un mayor plusvalor (Marx, 2004). Así, cada actividad económica va pasando de la cooperación simple a la manufactura y, por último, a la gran industria. En las dos últimas formas, la productividad incrementa por la vía de la división del trabajo y la mecanización, respectivamente. La manufactura reemplaza a la cooperación simple cuando el capital divide el trabajo de los artesanos y especializa a cada uno en una tarea parcial, lo que permite aumentar la productividad del trabajo. La base técnica de la manufactura continúa siendo subjetiva, ya que el trabajo manual persiste. El último cambio que realiza el capital sobre el proceso de trabajo consiste en la revolución de los medios de trabajo, mediante la conformación de un sistema de máquinas que constituye la gran industria. La base técnica de este proceso de trabajo es objetiva y tiende a incrementarse la composición orgánica del capital: el capital constante gana lugar frente al variable. Es decir, los requerimientos de fuerza de trabajo disminuyen, por lo que aquellas ramas donde prima la gran industria tienden a tener una mayor composición orgánica que aquellas donde prima la manufactura y, por tanto, una menor cantidad de trabajadores en relación a la producción. Por ello la cantidad de trabajadores ocupados no depende exclusivamente de los volúmenes de producción, sino también de la forma en que se organiza el proceso de trabajo.

En general, las actividades de la industria urbana suelen ser más mecanizadas que aquellas del sector servicios, que merced a su menor mecanización, utilizan una mayor proporción de fuerza de trabajo. Por el contrario, las actividades primarias, en especial la principal, la del cereal y las industriales, tienden a presentar una mayor composición orgánica del capital, por lo que el requerimiento de fuerza de trabajo es menor.

En el gráfico 1, se observa que el porcentaje de la producción y del empleo del sector industrial sobre el total de la economía no siguen una misma evolución. Si bien ambos tienden a caer, el porcentaje de asalariados industriales sobre el conjunto de asalariados disminuye en mayor medida que el porcentaje del PBI industrial sobre el total del PBI.

Figura 1. Porcentaje de PBI Industrial sobre PBI total y porcentaje de asalariados industriales sobre el total de asalariados, 1980-2005.

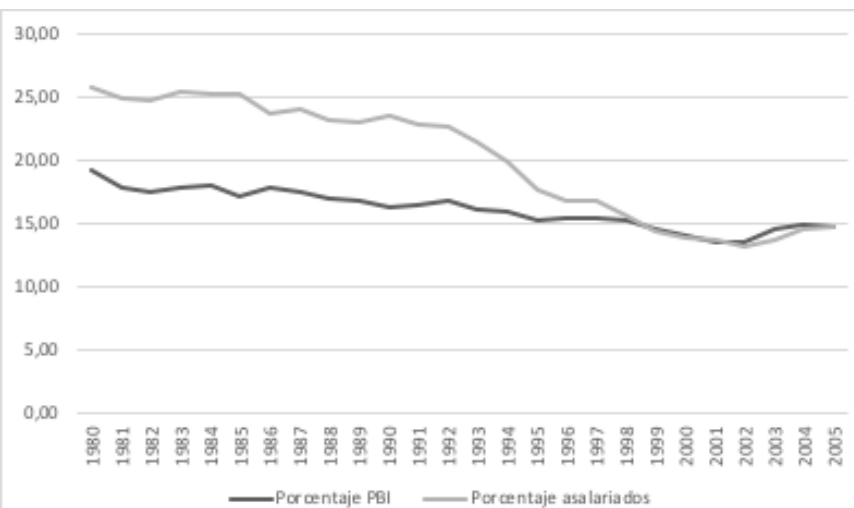

Fuente: elaboración propia con base en EPH y Cuentas Nacionales, Indec.

Si en el conjunto del período el PBI industrial disminuyó su participación en un 4,5\%, el trabajo industrial lo hizo en un $11 \%$. Ambas variables siguen una trayectoria similar durante la década del ochenta, pero a comienzos de los noventa comienza una caída más pronunciada del porcentaje de asalariados industriales. Ello podría explicarse por el proceso que describimos, en el que el aumento de la productividad determina una menor proporción de obreros por unidad de producto. Otra forma de observar este proceso es mediante el índice de producción física y de obreros ocupados en la industria (figura 2). 
Figura 2. Índice volumen físico de producción y nivel de ocupación industrial, 1997-2017, (1997=100).

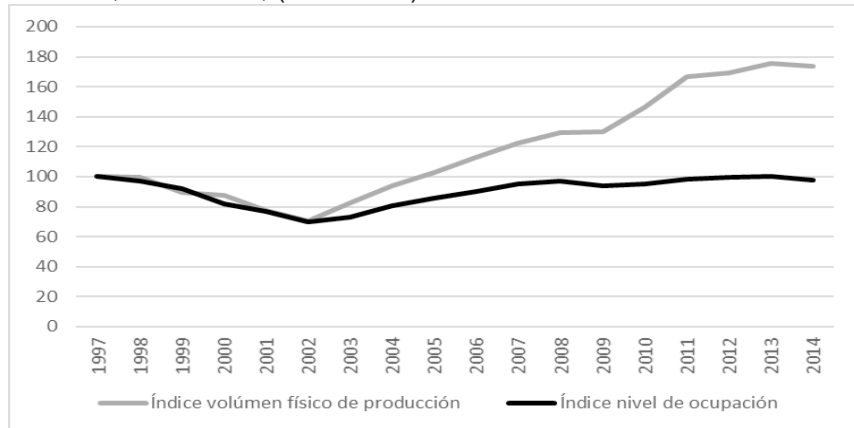

Fuente: elaboración propia con base en Encuesta Industrial Mensual, Indec.

Para ejemplificar, se puede observar cómo en la industria automotriz la incorporación de nuevas tecnologías a partir de la década del ochenta -como el control numérico y la robótica- permitió aumentar la productividad del trabajo (Chudnovsky, 1985; Lazcano, Menendez y Vocos, 1999; Fernández, 1998). Ello supuso un incremento de la producción a la par de una reducción de la cantidad de obreros empleados, como se observa en la tabla 3.

Tabla 3. Producción, personal ocupado y autos por obrero, sector automotriz, 1975 y 2014.

\begin{tabular}{lccc}
\hline & Producción & Personal ocupado & Autos por obrero \\
\hline 1975 & 240.036 & 54.556 & 4,40 \\
\hline 2014 & 617.329 & 33.232 & 18,58 \\
\hline Diferencia & 377.293 & -21.324 & 14,18 \\
\hline $\begin{array}{l}\text { Diferencia } \\
\text { porcentual }\end{array}$ & 157,18 & $-39,09$ & 322,21 \\
\hline
\end{tabular}

Fuente: elaboración propia con base en Asociación de Fabricantes de Automotores (2017)

En un plazo mayor, se observa claramente la evolución negativa del peso de las actividades industriales en la estructura ocupacional en relación a otras actividades, como se observa en la figura 3. Ello muestra que la disminución relativa de la cantidad de trabajadores en la industria no parece ser producto de una política económica específica, sino una tendencia de largo plazo que se explica por la forma en se desarrolla la producción bajo el capitalismo.
Figura 3. Porcentaje de asalariados industriales sobre el total de asalariados, 1950-2015.

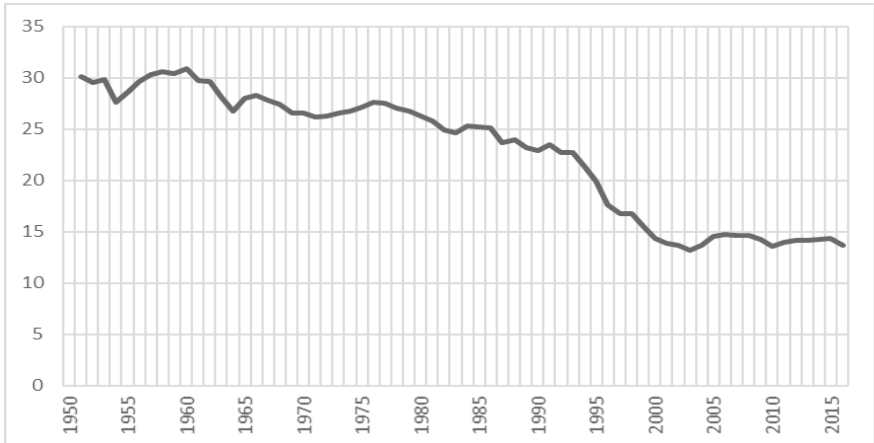

Fuente: medición propia con base en Kennedy (2011) y Cuentas Nacionales, Indec.

Una segunda distinción general que puede hacerse en la ocupación por sectores es si se trata del ámbito privado o estatal. Según la EPH, el $25 \%$ de los obreros son ocupados por el Estado (el censo de 2010 arroja un porcentaje mayor, del $28 \%$ ), mientras que el $75 \%$, en el sector privado. Como se aprecia en la figura 4, durante el período de los gobiernos kirchneristas, el porcentaje de asalariados estatales sobre el total de ocupados disminuye hasta 2007 y luego comienza una trayectoria ascendente, superando el nivel inicial.

Figura 4. Empleo estatal sobre el total de ocupados: 2003- 2015

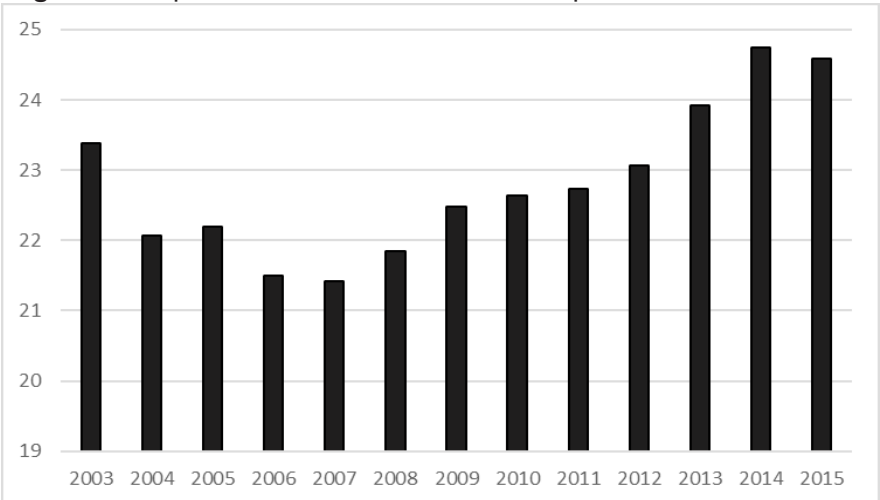

Fuente: elaboración propia con base en EPH

El empleo estatal cobra mayor peso en algunas provincias, donde alcanza porcentajes muy elevados. Por ejemplo, representa el 36\% de los ocupados en Río Gallegos; el $33,5 \%$ en Viedma; el 22\% en Gran La Plata; y el 24\% en Posadas.

Históricamente, el empleo estatal ha tendido a incrementarse en la Argentina. En el gráfico 5 se observa cómo se ha incrementado desde un 15\% en 1950 al 25\% de 2015, con picos a partir de 1989 que tienden a coincidir con los períodos de crisis. Ese año se sitúo en un $24 \%$ y en 
las décadas siguientes oscila entre $20 \%$ y $25 \%$.

Figura 5. Porcentaje de asalariados estatales sobre total de asalariados, 1950-2015

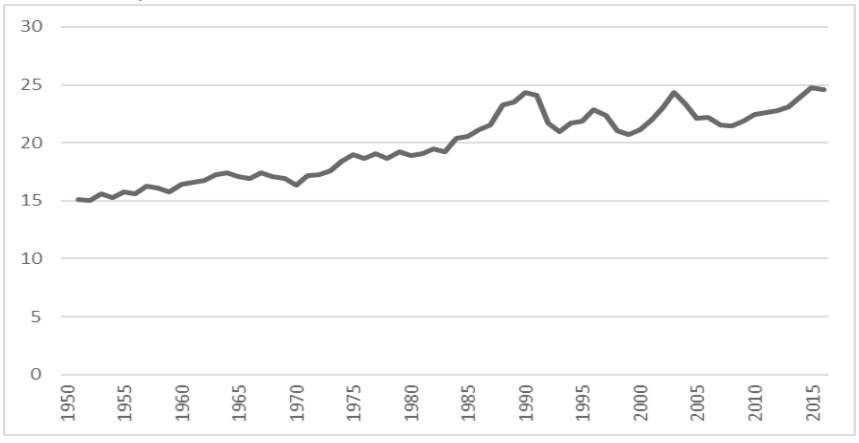

Fuente: Elaboración propia con base a Banco Central de la República Argentina (1975) y Fundación de Investigaciones Económicas Latinoamericanas (2015)

\section{El trabajo no registrado}

El trabajo no registrado es un elemento de degradación de las condiciones contractuales y salariales, además de una variable de fragmentación del conjunto de los trabajadores. En el largo plazo se verifica una tendencia al incremento del trabajo no registrado (Ilustración 6).

Figura 6. Tasa anual de empleo no registrado. Total de aglomerados, 1974-2015.

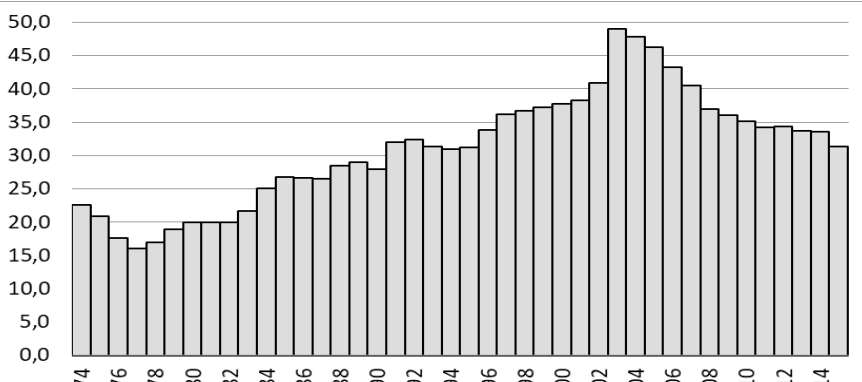

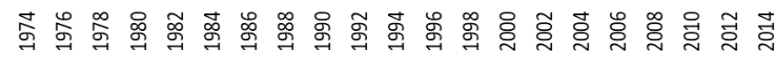

Fuente: Elaboración propia con base en EPH

Observamos un pico en la crisis de 2001, tras la cual se produce un descenso y se retorna al nivel previo. En comparación al período 2001-2002, la evolución posterior muestra una tendencia decreciente, por lo menos hasta el 2011. Pero a partir de ese año, se produce un estancamiento en torno al 34\%, y solo en 2015 se observa una caída al 31\%. Sin embargo, esta disminución del empleo no registrado no alcanzó a revertir los niveles de la década del 90.

Como explicamos, el kirchnerismo buscó reducir el trabajo no registrado mediante varias iniciativas. Como vemos en el gráfico 7 , si se compara el promedio de trabajo en negro durante los gobiernos kirchneristas con el promedio de los gobiernos menemistas, encontramos que se encuentra al mismo nivel. Es decir, se revirtió el nivel de la crisis de 2001, pero no el incremento previo.

Figura 7. Promedio de trabajo en negro por gobierno, 1976-2015

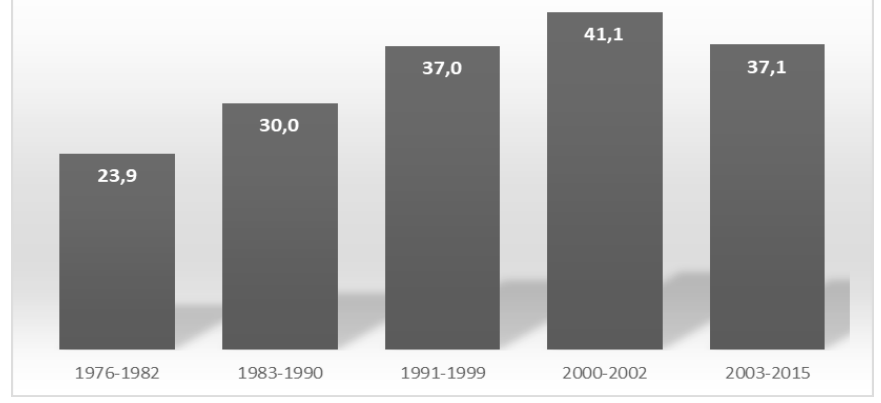

Fuente: Elaboración propia con base en EPH.

A la par del incremento tendencial del trabajo no registrado, observamos que la diferencia entre el salario de los obreros registrados y los no registrados tiende a aumentar. Es decir que los salarios de los trabajadores no registrados van descendiendo en relación a los registrados (figura 8).

Figura 8. Diferencia salarial entre trabajadores registrados y no registrados en porcentaje, 1974-2015.

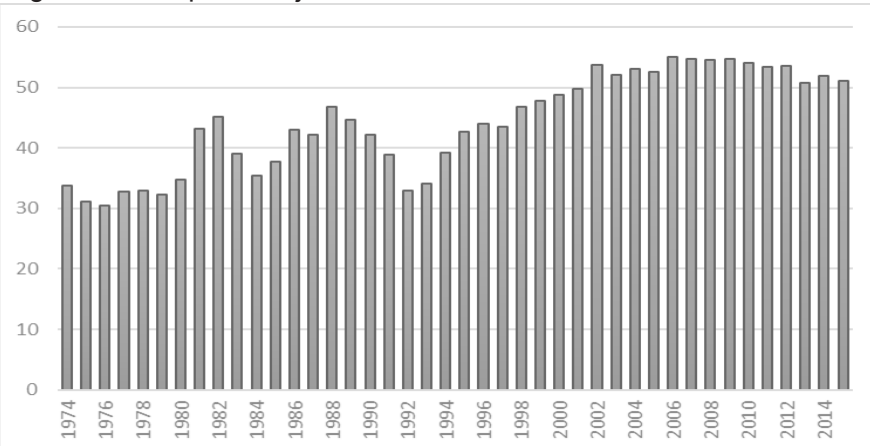

Fuente: Elaboración propia con base en EPH-Indec, IPC-GBA e IPC San Luis.

En 1974 los trabajadores no registrados percibían en promedio un $34 \%$ menos que los registrados, mientras que en 2015 esa diferencia fue de $51 \%$, es decir que, en cuatro décadas, la brecha incrementó un $17 \%$. La diferencia entre el nivel salarial de uno y otro sector fue creciendo de manera continua con un breve período de descenso entre 1989 y 1994, durante la primera presidencia de Menem. Durante el kirchnerismo se produce un leve aumento del $3 \%$ entre 2003 y 2009 y luego vuelve a descender un $4 \%$ hasta 2015 , ubicándose un $1 \%$ por debajo de 2003, pero 
aun por encima de los guarismos de la década de los 90 . Esta tendencia muestra que el trabajo no registrado no solo priva a los trabajadores de aportes a la seguridad social, sino que los coloca en un nivel salarial por debajo de los registrados.

El trabajo no registrado se concentra en los establecimientos de menor tamaño. El $74,3 \%$ de los obreros ocupados se emplea en establecimientos de hasta 100 trabajadores, como puede verse en la tabla 4 . Es decir que la concentración de trabajadores en grandes establecimientos no es un fenómeno extendido, si bien puede observarse una leve tendencia a una mayor concentración en establecimientos más grandes entre 2004 y 2015.

Tabla 4. Distribución de asalariados por tamaño de establecimiento, 2004 y 2015.

\begin{tabular}{cccccc}
\hline & $\begin{array}{c}\text { Hasta 10 } \\
\text { personas }\end{array}$ & $\begin{array}{c}\text { De 11 a } \\
\mathbf{4 0}\end{array}$ & $\begin{array}{c}\text { De 41 a } \\
\mathbf{1 0 0}\end{array}$ & $\begin{array}{c}\text { Más de } \\
\mathbf{1 0 0}\end{array}$ & TOTAL \\
\hline $\mathbf{2 0 0 4}$ & 52,25 & 17,90 & 10,06 & 19,79 & 100,00 \\
\hline $\mathbf{2 0 1 5}$ & 44,82 & 18,96 & 10,61 & 25,61 & 100,00 \\
\hline
\end{tabular}

Fuente: elaboración propia con base en EPH-Indec.

Las empresas pequeñas y medianas son las que emplean un mayor porcentaje de trabajadores no registrados. Como se ve en la tabla 5, en 2015 el empleo no registrado se concentró en un $83.9 \%$ en establecimientos de entre 1 y 10 empleados. Los guarismos de estos años no se han modificado significativamente respecto de los de 2004, lo que indica que la política que fomentó el registro de trabajadores destinada especialmente a las pequeñas y medianas empresas no rindió frutos.

Tabla 5. Distribución de asalariados por tamaño de establecimiento según condición de registro, en porcentajes, 2004 y 2015.

\begin{tabular}{clccccc}
\hline \multirow{2}{*}{2004} & $\begin{array}{c}\text { Hasta 10 } \\
\text { personas }\end{array}$ & $\begin{array}{c}\text { De 11 a } \\
40\end{array}$ & $\begin{array}{c}\text { De 41 a } \\
100\end{array}$ & $\begin{array}{c}\text { Más } \\
\text { de 100 }\end{array}$ & TOTAL \\
\cline { 2 - 7 } & $\begin{array}{l}\text { Registrados } \\
\text { registrados }\end{array}$ & 23,90 & 25,70 & 16,53 & 33,86 & 100,00 \\
\hline \multirow{2}{*}{2015} & 84,16 & 9,12 & 2,78 & 3,94 & 100,00 \\
\cline { 2 - 7 } & $\begin{array}{l}\text { Registrados } \\
\text { Nogistrados }\end{array}$ & 23,95 & 23,85 & 14,96 & 37,23 & 100,00 \\
\hline
\end{tabular}

Fuente: elaboración propia con base en EPH-INDEC.

*La variable descuentos o aportes se refiere a las cargas sociales y es el indicador de trabajo registrado.

Dado que el trabajo no registrado está fuertemente asociado a la presencia de establecimientos de menor tamaño, no es casual entonces que las ramas con mayor porcentaje de trabajo no registrado tengan un porcentaje elevado de trabajadores ocupados en establecimientos pequeños y medianos (tabla 6).

Tabla 6. Porcentaje de empleo registrado y no registrado y distribución por tamaño de establecimiento, ramas seleccionadas, 2015.

\begin{tabular}{|c|c|c|c|c|c|c|c|c|}
\hline \multirow[b]{2}{*}{ Rama } & \multirow[b]{2}{*}{$\begin{array}{l}\text { Regis- } \\
\text { trados }\end{array}$} & \multirow{2}{*}{$\begin{array}{l}\text { No. } \\
\text { regis- } \\
\text { tra- } \\
\text { dos }\end{array}$} & \multicolumn{6}{|c|}{ Tamaño del establecimiento } \\
\hline & & & 0.0 & $\begin{array}{l}\text { De } 1 \\
\text { a } 10\end{array}$ & $\begin{array}{c}\text { De } 11 \\
\text { a } 40\end{array}$ & $\begin{array}{l}\text { De } 41 \\
\text { a } 110\end{array}$ & $\begin{array}{c}\mathrm{De} \\
101 \mathrm{a} \\
500\end{array}$ & $>500$ \\
\hline $\begin{array}{l}\text { Silvicultura, } \\
\text { extracción } \\
\text { de madera y } \\
\text { actividades } \\
\text { de apoyo. }\end{array}$ & 9.2 & 90.8 & 0.0 & 84.96 & 15.04 & 0.0 & 0.0 & 0.0 \\
\hline $\begin{array}{l}\text { Venta al } \\
\text { por menor } \\
\text { en puestos } \\
\text { móviles y no } \\
\text { realizadas } \\
\text { en tiendas } \\
\text { n.c.p. }\end{array}$ & 13.8 & 85.1 & 0.0 & 35.59 & 5.86 & 2.03 & 4.71 & 51.8 \\
\hline $\begin{array}{l}\text { Activida- } \\
\text { des de los } \\
\text { hogares } \\
\text { como em- } \\
\text { pleadores } \\
\text { de personal } \\
\text { doméstico. }\end{array}$ & 20.2 & 79.8 & 99.25 & 0.58 & 0.0 & 0.0 & 0.0 & 0.17 \\
\hline $\begin{array}{l}\text { Manteni- } \\
\text { miento y } \\
\text { reparación } \\
\text { de vehículos } \\
\text { automoto- } \\
\text { res, excepto } \\
\text { motocicle- } \\
\text { tas. }\end{array}$ & 27.1 & 72.9 & 0.0 & 86.58 & 1.68 & 0.0 & 0.0 & 11.74 \\
\hline $\begin{array}{l}\text { Fabricación } \\
\text { de vehículos } \\
\text { automo- } \\
\text { tores, } \\
\text { remolques y } \\
\text { semirremol- } \\
\text { ques. }\end{array}$ & 95.9 & 3.0 & 0.0 & 7.04 & 1.46 & 6.34 & 20.59 & 64.57 \\
\hline $\begin{array}{l}\text { Fabricación } \\
\text { de pinturas, } \\
\text { barnices y } \\
\text { productos } \\
\text { de reves- } \\
\text { timiento } \\
\text { similares, } \\
\text { tintas de } \\
\text { imprenta y } \\
\text { masilla. }\end{array}$ & 94.0 & 6.0 & 0.0 & 0 & 5.29 & 11.76 & 13.59 & 69.37 \\
\hline $\begin{array}{l}\text { Pompas } \\
\text { fúnebres } \\
\text { y servicios } \\
\text { conexos. }\end{array}$ & 94.7 & 5.3 & 0 & 37.11 & 20.88 & 4.58 & 0.0 & 37.43 \\
\hline $\begin{array}{l}\text { Fabricación } \\
\text { de produc- } \\
\text { tos farma- } \\
\text { céuticos, } \\
\text { sustancias } \\
\text { químicas } \\
\text { medicinales } \\
\text { y productos } \\
\text { botánicos. }\end{array}$ & 94.8 & 5.2 & 0 & 4.55 & 12.78 & 4.26 & 38.52 & 39.89 \\
\hline $\begin{array}{l}\text { Fabricación } \\
\text { de papel y } \\
\text { productos } \\
\text { de papel. }\end{array}$ & 89.0 & 11.0 & 0 & 11.96 & 6.7 & 24.37 & 10.72 & 46.24 \\
\hline
\end{tabular}

Fuente: elaboración propia con base en EPH 
Como ejemplo, una de las actividades con mayor incidencia de trabajo no registrado es la extracción de madera $(90,8 \%)$. Allí el $84,96 \%$ de los obreros se emplean en establecimientos de hasta 10 trabajadores. Otros sectores con un porcentaje elevado de trabajo no registrado son Comercio (por ejemplo: ventas minoristas, $85 \%$, y talleres de reparación, 73\%) y Gastronomía (con un promedio del $54 \%$ ). Ambas ramas se caracterizan por la presencia de establecimientos pequeños. En Comercio, por ejemplo, el $51 \%$ de los trabajadores se emplea en establecimientos de hasta 10 obreros y en Gastronomía lo hace el 48\%. En Construcción, el 59\% de los trabajadores se ocupa en obras con menos de diez empleados y el trabajo no registrado alcanza un $68,3 \%$. Una situación similar se encuentra en el empleo doméstico, que tiene un $79,8 \%$ de trabajo no registrado.

Por el contrario, las ramas donde hay mayor presencia de trabajo registrado tienden a ser aquellas donde existe una mayor concentración de trabajadores, en establecimientos de mayor tamaño. Por ejemplo, en la industria manufacturera, encontramos la fabricación de pinturas con un $94 \%$ de empleo registrado y un $82 \%$ de trabajadores en establecimientos de más de 100 empleados. La industria manufacturera es un sector que ha mostrado una mayor concentración de ocupados en empresas de mayor tamaño (Tabla 7). El análisis de distribución por tamaño de establecimiento arroja que, en promedio, se trata de un sector donde se da una gran concentración en empresas de más de 500 trabajadores, un $38,44 \%$, y que un $58 \%$ se emplea en empresas de más de 100 empleados. Al interior de este sector, encontramos ramas de mayor nivel de concentración como elaboración de productos de tabaco, donde el $95,78 \%$ se emplea en establecimientos de más de 500 trabajadores, y otras de menor como la fabricación de muebles, donde el 61,75\% se ubica en empresas de hasta 10 trabajadores.

Tabla 7. Distribución porcentual de los trabajadores de la industria manufacturera, promedio de las 40 ramas, según tamaño del establecimiento, 2015.

\begin{tabular}{cc}
\hline Tamaño del establecimiento & Porcentaje \\
\hline De 1 a 10 & 19,92 \\
\hline De 11 a 40 & 15,59 \\
\hline De 41 a 100 & 6,45 \\
\hline De 101 a 500 & 19,60 \\
\hline Más de 500 & 38,44
\end{tabular}

Fuente: elaboración propia con base a EPH

\section{La curva de los salarios}

El nivel salarial constituye la variable más palpable de la evolución de las condiciones materiales de la clase obrera. Como observamos en la ilustración 9, existe una tendencia a la caída del salario real que se verifica desde mediados de la década del 70. Entre 1974 y 2015 el promedio salarial de los trabajadores ocupados descendió un $47 \%$. Es decir, se ubica prácticamente en la mitad. Desde comienzos de los 90, el nivel salarial ha tendido a mantenerse dentro de una misma franja, con una caía pronunciada entre 1999 y 2003 , del $31 \%$, tras lo cual vuelve a recuperarse lentamente para alcanzar en 2009 el nivel de 1993, el mayor hasta ese momento. Luego se produce un ascenso del 10\% hasta 2013, tras el cual comienza una nueva caída del 13\% hasta 2015.

Figura 9. Evolución del salario promedio del total de asalariados, 1974-2015

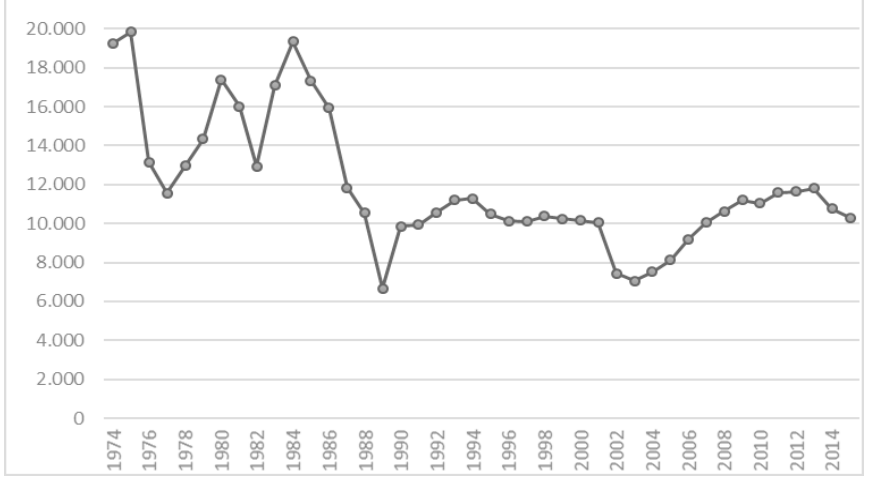

Fuente: elaboración propia con base en EPH-INDEC, IPC-GBA e IPC San Luis.

Dadas la variabilidad de la evolución salarial durante los períodos de gobierno, el promedio salarial de cada etapa nos permite evaluar en qué nivel en términos históricos se situaron las remuneraciones a los asalariados (ilustración 10). Así, observamos que el gobierno de Alfonsín mantuvo un nivel similar al de la dictadura militar, mientas los mandatos de Menem llevaron los salarios un escalón más abajo. Luego, los gobiernos de De la Rúa y Duhalde lo hicieron descender aún más. Los gobiernos kirchneristas recuperaron el nivel salarial, pero lo dejaron levemente por debajo del nivel del menemismo. 
Figura 10. Promedio salarial del total de asalariados, por gobierno 1974, 2015.

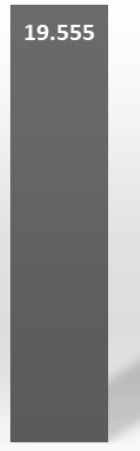

1974-1975
$1976-1982$

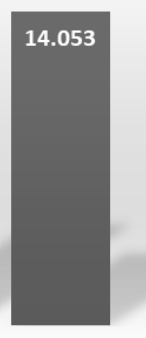

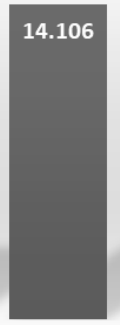

1983-1989

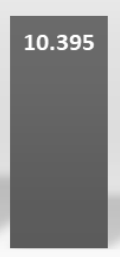

1990-1999

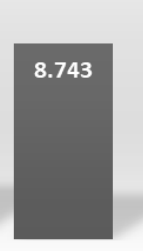

2000-2002

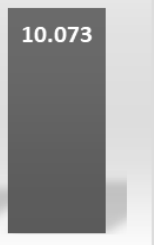

2003-2015
Fuente: elaboración propia con base en EPH-INDEC, IPC-GBA e IPC San Luis.

Resulta interesante señalar que aquellos sectores que tienen peores niveles salariales cuentan con una participación importante en el total del empleo. Si tomamos los salarios de 2015, según la EPH, vemos que los peores salarios se encuentran en Servicio doméstico (\$1.964), Gastronomía (\$4.118) y Construcción (\$4.297).

\section{La precarización contractual}

Otra vía de degradación de las condiciones laborales es el contrato por tiempo determinado, que representa el 9\% del total de los asalariados en 2015. Este tipo de contrato implica una situación de estabilidad laboral más precaria respecto al contrato por tiempo indefinido, que supone además el pago de una indemnización en caso de que el empleador decida extinguirlo. Es por este motivo que describimos el proceso de aumento de este tipo de contratos como precarización contractual.

En servicios de expendio de comidas y bebidas en puestos ambulantes, construcción y en cultivos agrícolas es donde mayor porcentaje hay de contratos por tiempo determinado ( $46 \%, 36 \%$ y $38 \%$ respectivamente), por las características de estas ramas (tabla 8). Pero este tipo de contratación también tiene una incidencia alta en otras ramas como fabricación de calzado (19\%), recolección de residuos (20\%), consultorías de gestión empresarial (21\%), veterinaria $(21 \%)$, entre otras. El sector agrícola cuenta con una cantidad muy alta de trabajadores temporarios, por la naturaleza de la actividad. Si se toman los trabajadores registrados en la Seguridad Social, el 68\% corresponde a trabajadores permanentes, el $17 \%$ es no permanente, el $14 \%$ de temporada. Los trabajadores no permanentes se concentran en las actividades de cultivo de cereales (15\%), cultivo de tabaco (11\%) y servicios agrícolas (11\%). Los convenios de corresponsabilidad gremial han cobrado cierta importancia en el cultivo de vid, abarcando el 9,3\% de los trabajadores registrados y en la actividad forestal, donde alcanzó al 9,6\%. En menor medida se encuentra en el cultivo de algodón (4\%). Ello no parece haber repercutido positivamente en los niveles de empleo no registrado.

Tabla 8. Porcentaje de contratos por tiempo determinado e indeterminado, ramas seleccionadas, 2015.

\begin{tabular}{lcc}
\hline & $\begin{array}{c}\text { Contrato tiempo } \\
\text { determinado }\end{array}$ & $\begin{array}{c}\text { Contrato tiempo } \\
\text { indeterminado }\end{array}$ \\
\hline Construcción & $36,77 \%$ & $54,23 \%$ \\
\hline Cultivos agrícolas & $38,72 \%$ & $51,24 \%$ \\
\hline $\begin{array}{l}\text { Fabricación de componentes } \\
\text { electrónicos }\end{array}$ & $14,11 \%$ & $85,89 \%$ \\
\hline Administración pública & $10,28 \%$ & $84,49 \%$ \\
\hline
\end{tabular}

Fuente: elaboración propia con base en EPH

El empleo estatal ha sido un sector donde el crecimiento del empleo tuvo como base la expansión de los contratos a término y otros tipos de contratación precaria. Además del régimen de planta permanente, en el Estado existen otras modalidades de contratación como la planta transitoria, pasantías, becas, contratos de locación de servicio, de obra, con organismos internacionales, con universidades o fundaciones. En este sentido, durante la década kirchnerista se observa un aumento exponencial de los llamados contratados, es decir aquellos que no cuentan con estabilidad laboral. Por ejemplo, bajo la órbita del Poder Ejecutivo Nacional, el empleo público pasó de 241.383 trabajadores en el 2003 a 366.365 en 2015, un aumento del 51,78\%. De este total, los trabajadores de planta permanente y transitoria pasaron de 226.250 a 297.365 constituyendo un aumento del 31,43\%; mientras que, los contratados aumentaron de 15.133 a 69.000 , es decir, un crecimiento del $355 \%$. Con esta somera muestra se dimensiona el crecimiento de esta forma precaria de trabajo. En el seno de los estatales precarizados podemos distinguir, además, a quienes son empleados bajo la ley de pasantías y a los monotributistas. En el caso de los pasantes, no se abonan salarios sino una "asignación estímulo" ya que se considera que la pasantía educativa no origina ningún tipo de relación laboral entre el pasante y la empresa u organización en la que se realiza (Ley 26.427). De este modo, se utiliza fuerza 
de trabajo sin realizar las contraprestaciones comunes a los trabajadores que realizan la misma actividad y se cercena el derecho a la sindicalización. La contratación de monotributistas supone que el Estado los emplea para la realización de un "servicio", aunque muchas veces se trata de una relación de dependencia encubierta. Este tipo de contrato que se supone "independiente" exime al Estado de la responsabilidad de garantizar la estabilidad laboral. El 7\% de los trabajadores no percibió descuentos jubilatorios durante el año 2014. Ese porcentaje comprende a los monotributistas, ya que su carácter de trabajador independiente implica, entre otras cosas, que el Estado no realice aportes previsionales. Pero cuando clasificamos a ese segmento entre los que realizan aportes propios y los que no, encontramos que de ese 7\% sólo el $44 \%$ declara aportar por su cuenta. El 56\% restante constituiría trabajo no registrado dentro del Estado.

La evolución de las formas de contratación de las últimas décadas (ilustración 11) en el conjunto de la economía, muestra que la cantidad de asalariados con contrato por tiempo indeterminado tiende a disminuir, es decir que las formas contractuales precarias tienden a elevarse.

Figura 11. Porcentaje de asalariados con contrato por tiempo indeterminado sobre el total de asalariados*, 1995-2015.

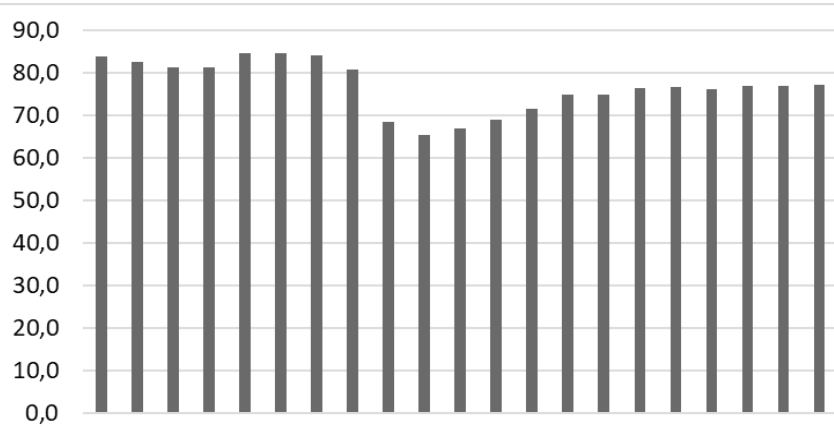

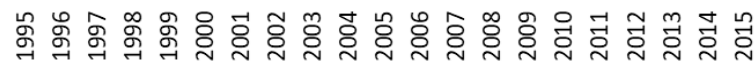

Fuente: elaboración propia con base en EPH.

* Se tomó la medición de esta variable, dado que hay un porcentaje importante de casos sin datos, dadas las dificultades que tiene la Encuesta Permanente de Hogares para captar situaciones

contractuales que no implican un contrato por tiempo indeterminado.

Se observa una caída importante entre 2003 y 2004, cuando pasa del $80,9 \%$ de 2002 a $68,7 \%$ en 2003 y $65,5 \%$ en 2004. Es decir que desciende un $15 \%$ en dos años. Luego comienza un leve ascenso que no recupera el nivel previo a la caída. Así, en 2015 se ubica en un 77,4\%.

\section{Evolución de la tasa de afiliación sindical}

Otro aspecto a tener en cuenta para evaluar la situación laboral es la tasa de afiliación sindical, que muestra el nivel de organización gremial de los trabajadores. El último dato disponible es de la Encuesta del Ministerio de Trabajo de 2008 (tabla 9) y muestra que la cantidad de afiliados a sindicatos representa solo el $37,7 \%$ de los trabajadores. Los sectores con mayor tasa de afiliación son transporte, almacenaje y comunicación, que pertenecen al gremio de camioneros, y construcción, con un $49,2 \%$ y $47,3 \%$ respectivamente. Los gremios industriales $(45,2 \%)$, comercio y gastronomía siguen en importancia $(42,3 \%)$. Con menor porcentaje se encuentran los servicios financieros $(25,4 \%)$.

Tabla 9. Porcentaje de afiliación sindical por rama (2008)

\begin{tabular}{ll}
\hline Industria manufacturera & 45,2 \\
\hline Construcción & 47,3 \\
\hline Comercio, restaurantes y hoteles & 42,3 \\
\hline Transporte, almacenaje y comunicaciones & 49,2 \\
\hline Servicios financieros y a las empresas & 25,4 \\
\hline Servicios comunales, sociales y personales & 24,3 \\
\hline Total & 37,7 \\
\hline
\end{tabular}

Fuente: encuesta del Ministerio de Trabajo.

Si se compara históricamente, la tasa de afiliación bajo el kirchnerismo se recuperó respecto a fines de los noventa, pero no superó la existente hasta mediados de esa década. En el 2000 esta tasa era de $31,7 \%$, pero en 1995, del 38,7\% y, si analizamos el largo plazo, en 1974, fue del $43 \%$. Según distintas mediciones (tabla 10), durante el kirchnerismo nos encontraríamos en niveles históricos de sindicalización bajos, a pesar del discurso de la "revitalización sindical". Las mediciones históricas que se han podido recopilar dan los siguientes resultados:

Tabla 10. Estimaciones de tasa de afiliación sindical, 1954-2008

\begin{tabular}{|c|c|c|}
\hline Año & Medición 1 & Medición 2 \\
\hline 1954 & $48 \%$ & \\
\hline 1963 & $40 \%$ & \\
\hline 1974 & $43 \%$ & \\
\hline 1979 & $42 \%$ & 67,5 \\
\hline 1982 & $41 \%$ & \\
\hline 1985 & & 65,6 \\
\hline 1989 & $44 \%$ & 38,7 \\
\hline 1990 & & 31,7 \\
\hline 1995 & & \\
\hline 2000 & & \\
\hline
\end{tabular}




\begin{tabular}{|c|c|c|}
\hline 2005 & 37 \\
\hline 2006 & & 39,7 \\
\hline 2008 & & 37 \\
\hline
\end{tabular}

Fuente:

Medición 1: Orsatti y Lamadrid (1999)

Medición 2: Senén, Trajtemberg, y Medwid (2010)

El empleo no registrado y el empleo en pymes explican parte del problema, pero la baja afiliación es registrada en las empresas más grandes, con mayor presencia del empleo registrado. A su vez, en el interior del país la tasa de afiliación tiende a ser mayor: $44 \%$ contra el $35,8 \%$ en el Gran Buenos Aires (GBA). Otro dato que muestra el bajo nivel de organización sindical, al menos en el sector privado, es la presencia de delegados: solo el $14,2 \%$ de las empresas cuenta con al menos un delegado gremial. Si se contabiliza la cantidad de obreros que se encuentran en empresas con representación gremial, se obtiene el 39\%. Es decir que hay cierta relación entre los obreros que se afilian y aquellos que tienen representación sindical en su lugar de trabajo.

\section{Conclusiones}

El Kirchnerismo ha implementado una serie de políticas laborales que intentaron modificar la estructura del mercado de trabajo. En especial, a disminuir el porcentaje de trabajo no registrado, tanto en el ámbito urbano como en el rural, prestando especial atención a las pymes. El análisis estadístico permitió abordar una serie de aspectos acerca de la estructura de la clase obrera argentina luego de los gobiernos kirchneristas. En primer lugar, la mayor parte de la clase obrera se emplea en el sector terciario, en especial el relacionado con los servicios. La industria manufacturera, en cambio, emplea un porcentaje menor, aunque ella se subdivide en un número elevado de ramas, y cada una de ellas emplea un porcentaje minúsculo del total de asalariados. Es de destacar que un porcentaje importante de trabajadores se encuentra en el sector de la construcción y en el empleo doméstico. Se trata de sectores con un elevado índice de informalidad laboral y de contratos por tiempo determinado. A ello se debería sumarle que se encuentran entre los sectores peores pagos.

Otro dato destacable es la cantidad de trabajadores empleados por el Estado. Se trata del 22\% del total, contabilizando los trabajadores de diferentes ramas. En cuanto al empleo en administración pública exclusivamente, se observa un porcentaje elevado, que en algunas provincias alcanza niveles por encima del $25 \%$, es decir, más de un cuarto de la población, lo cual resulta un dato sumamente llamativo, pero que da cuenta sobre cómo el empleo estatal parece haber crecido como forma de absorber a aquellas fracciones desocupadas y mantenerlas encubiertas como sobrepoblación relativa latente (Kabat, 2009).

En cuanto al sector agrario, a pesar de su importancia económica, emplea un porcentaje pequeño de trabajadores. Una característica del empleo agrario es la precariedad de las condiciones de los trabajadores, tanto por la presencia del trabajo no registrado como de las formas temporales de contratación. Como mencionamos, la estructura ocupacional no guarda relación directa con la estructura productiva, dado que el nivel de ocupación no solo está determinado por la cantidad de producción, sino por cómo se realiza la misma, particularmente por el proceso de trabajo. Como ejemplo, la industria manufacturera muestra que el aumento de la producción no va necesariamente acompañado del aumento de la ocupación en el sector, e incluso puede implicar su disminución cuando se aumenta la productividad. Así, vemos que el descenso histórico que sufrió el sector manufacturero en la estructura ocupacional no se explicaría por una disminución de la producción, sino que deben tenerse en cuenta los cambios productivos.

Hemos visto cómo existe una tendencia histórica al aumento del trabajo no registrado y cómo la misma no se ha revertido durante los gobiernos kirchneristas. Otro aspecto relevante que exploramos es la relación entre el trabajo no registrado y el tamaño de los establecimientos. Es decir, en qué medida el empleo en las llamadas pymes es responsable de la alta tasa de empleo no registrado. Efectivamente, lo que encontramos es que las pymes son responsables por la mayor parte del trabajo en la Argentina. Incluso en aquellas ramas con un menor nivel de centralización de capital son aquellas donde encontramos mayores índices de trabajo no registrado. Este dato indica que la subsistencia de las pequeñas y medianas empresas depende en gran medida de la disminución sus costos laborales por la vía del empleo no registrado, lo cual les permite también no cumplir con la legislación laboral ni los convenios colectivos. Ello puede verificarse también al comparar la diferencia salarial entre los trabajadores registrados y los no registrados, que tiende a aumentar.

El nivel salarial muestra, en consonancia con otras 
variables, una tendencia histórica al deterioro. Durante el período de los gobiernos kirchneristas se observa una recuperación respecto de la caída inmediatamente anterior producida por la crisis de 2001, que ubica a los salarios levemente por debajo del nivel de la década del 90. Pero hacia el final del período se produce una nueva caída.

Un último aspecto que se aborda es la tasa de afiliación. Durante los últimos doce años no se ha revertido la tendencia descendente de la misma, a pesar del discurso sobre la revitalización sindical. Explicar esta tendencia requeriría una indagación particular, pero es importante señalar que no se trata de una tendencia propia de Argentina, sino que se repite a nivel mundial (Visser, 2015).

Sin embargo, resulta un dato significativo que frente a la degradación de las condiciones laborales, la clase obrera ocupada no recurra a los sindicatos como forma de organización. Estos datos ponen en cuestión la imagen que presenta a las políticas de trabajo de los gobiernos kichneristas como responsabilidad de una recuperación de la calidad del empleo y del protagonismo de los sindicatos. Por el contrario, consideramos que las mismas no han revertido tendencias de más largo plazo que operan en la Argentina y que conllevan una mayor fragmentación de la clase obrera y precariedad del empleo.

\section{Referencias}

1. Asociación de Fabricantes de Automotores (2017). Anuario estadístico. Disponible en: https://goo.gl/ ugsh9d

2. Atzeni, M. y Ghigliani, P. (2008). Nature and limits of trade unions mobilizations in contemporary Argentina. LabourAgain Publications on Latin America, IISG, Amsterdam. Publicación en línea disponible en: http://www.iisg.nl/labouragain/ documents/atzeni-ghigliani.pdf

3. Azpiazu, D. y Schorr, M. (2010). La industria argentina en la posconvertibilidad: reactivación y legados del neoliberalismo. Problemas del desarrollo, 41(161), 111-138.

4. Banco Central de la República Argentina (1975). Sistema de cuentas del producto e ingreso de la
Argentina. Volumen 2. Buenos Aires.

5. Beccaria, L. y Maurizio, R. (2012). Reversión y continuidades bajo dos regímenes macroeconómicos diferentes. Mercado de trabajo e ingresos en argentina 1990-2010. Desarrollo económico, 52(206), 205-228.

6. Bekerman, M. y Vázquez, D. (2016). Principales transformaciones de la estructura industrial argentina durante la posconvertibilidad. Ensayos de Economía, 26(48), 35-65.

7. Chudnovsky, D. (1985). La difusión de tecnología de punta en la Argentina: el caso de las máquinas herramientas con control numérico, el CAD/CAM y los robots. Desarrollo económico, (96), 483-515.

8. Fernández Bugna, C. y Porta, F. (2008). El crecimiento reciente de la industria Argentina. Nuevo régimen sin cambio estructural. Realidad Económica, 233, 17-48.

9. Fernández, F. (1998). Cambios en los procesos de trabajo en la industria actual: el caso de la industria automotriz. Pimsa. Documento de trabajo No. 11. Disponible en: http://www.pimsa.secyt.gov.ar/ publicaciones/DT11.pdf

10. Fundación de Investigaciones Económicas Latinoamericanas (2015). Evolución del empleo público. Argentina. Disponible en: https://goo.gl/ $\mathrm{qNgyV}$

11. Kabat, M. (2009). La sobrepoblación relativa. El aspecto menos conocido de la concepción marxista de la clase obrera. Anuario CEICS, 3, 109-128.

12. Kennedy, D. (2011). Economía política de la contabilidad social. Vínculos entre la teoría de la riqueza social y sus formas de cuantificación. Tesis doctoral. Universidad de Buenos Aires.

13. Lascano, V., Menendez, F., y Vocos, F. (1999). Análisis del proceso de trabajo en la planta de automóviles Ford. Disponible en: https://goo.gl/ qqApfb.

14. Marx, Karl. (2004): El capital, siglo XXI, Buenos Aires, Tomo I. 
15. Orsatti, A. y Lamadrid, A. (1999). Una revisión de las medidas sobre la tasa de sindicalización en Argentina. Estudios del Trabajo, 2, 135-159.

16. Palomino, H. (2008). La instalación de un Nuevo Régimen de Empleo en Argentina: de la Precarización a la regulación. Revista Latinoamericana de Estudios del Trabajo - RELET, 12(19), 121-144.

17. Palomino, H. y Dalle, P. (2012). El impacto de los cambios ocupacionales en la estructura social de la Argentina: 2003-2011. Revista de trabajo, 8(10), 205-223.

18. Panigo, D. y Neffa, J.C. (2009). El mercado de trabajo argentino en el nuevo modelo de desarrollo. Documento de trabajo. Secretaria de Política Económica. Subsecretaria de Programación Económica. Disponible en: https://goo.gl/n73H9h
19. Salvia, A. y Gutiérrez Ageitos, P. (2013). La estructura social del trabajo en Argentina en el cambio de siglo: cuando lo nuevo no termina de nacer. Papeles de población, 19(76), 163-200.

20. Senén González, C., Trajtemberg, D. y Medwid, B. (2010). Tendencias actuales de la afiliación sindical en Argentina: evidencias de una encuesta a empresas. Relations industrielles, 65(1), 30-51.

21. Visser, J. (2015). Data Base on Institutional Characteristics of Trade Unions, Wage Setting, State Intervention and Social Pacts, 1960-2014 (ICTWSS), disponible en: https://goo.gl/c4vfhD. 